\title{
The effect of DCIS grade on rate, type and time to recurrence after 15 years of follow-up of screen-detected DCIS
}

\begin{abstract}
MG Wallis*,I, K Clements ${ }^{2}$, O Kearins ${ }^{2}$, G Ball ${ }^{3}$, J Macartney ${ }^{2}$ and GM Lawrence ${ }^{4}$
'Cambridge Breast Unit and NIHR Cambridge Biomedical Research Unit, Box 97, Cambridge University Hospitals NHS Foundation Trust, Hills Road, Cambridge CB2 OQQ, UK; ${ }^{2}$ West Midlands QA Reference Centre, West Midlands Cancer Intelligence Unit, Public Health Building, University of Birmingham, Birmingham BI5 2TT, UK; ${ }^{3}$ John van Geest Cancer Research Centre, School of Science and Technology, Nottingham Trent University, Clifton Lane, Nottingham NGII 8NS, UK; ${ }^{4}$ West Midlands Cancer Intelligence Unit, Public Health Building, University of Birmingham, Birmingham B 5 $2 \pi$, UK
\end{abstract}

BACKGROUND: The incidence of ductal carcinoma in situ (DCIS) rose rapidly when the NHS Breast Screening Programme (NHSBSP) started in 1988. Some authorities consider that this represents both over-diagnosis and over-treatment. We report long-term follow-up of DCIS diagnosed in the first 10 years (April 1988 to March 1999) of the West Midlands NHSBSP.

METHODS: 840 noninvasive breast cancers were recorded on the national breast screening computer system. Following exclusions, and thorough case note and pathology review, 700 DCIS cases were identified for follow-up.

RESULTS: After a median follow-up of I 83 (range I 33 to 259) months, I02 (I4.6\%) first local recurrences were identified, 49 (48\%) were invasive. Median time to first noninvasive recurrence was 15 months, and 60 months for invasive recurrence. Median time to invasive recurrence was 76 months from initially high-grade DCIS, and I 31 months from low/intermediate grade DCIS. For the seven women, presenting with metastasis as their first event, the median time was 82 (range 15 to 188) months. The cumulative proportion developing recurrence at 180 months was twice as high as at 60 months.

INTERPRETATION: Short-term follow-up of patients diagnosed with DCIS will miss significant numbers of events, especially invasive local recurrences.

British Journal of Cancer (2012) 106, 1611-1617. doi:10.1038/bjc.2012.151 www.bjcancer.com

Published online 19 April 2012

(C) 2012 Cancer Research UK

Keywords: ductal carcinoma in situ; DCIS; follow-up; breast cancer

Since the introduction of the UK National Health Service Breast Screening Programme (NHSBSP) in 1988, there has been a large increase in the incidence of ductal carcinoma in situ (DCIS). Thus, in the year before the introduction of screening (ONS, 1998), 295 cases of DCIS were recorded in England and Wales, in women aged 50-64, while 2500 cases were detected, by screening, in the year 2008/2009 (NHSBSP and ABS, 2010), DCIS now accounts for around $20 \%$ of screen-detected breast cancers (Evans et al, 2001, NHSBSP and ABS, 2010; Virnig et al, 2010). One of the main reasons for this increase is that DCIS is relatively easy to detect on a mammogram owing to the micro-calcification that is frequently present (Morrow et al, 2002). Fewer cases of DCIS present symptomatically as about $80 \%$ of cases are nonpalpable and diagnosed by mammography alone (Mokbel and Cutuli, 2006; Virnig et al, 2010). However, it is worthy of note that, in 2006, in England, $40 \%$ of DCIS presented outside the national screening programme (NCIN and NHSBSP, 2009). The increase in the incidence of DCIS and the uncertainty about its propensity to become malignant, if left untreated, have led to discussions about overdiagnosis (Welch et al, 2008; Jørgensen and Gøtzsche, 2010). These are not just confined to noninvasive breast cancer, and the

\footnotetext{
*Correspondence: Dr MG Wallis;

E-mail: matthew.wallis@addenbrookes.nhs.uk

Received 22 November 2011; revised 24 February 2012; accepted 22

March 2012; published online 19 April 2012
}

arguments about overdiagnosis in screening, in general (Zahl et al, 2004; Duffy et al, 2010; Jørgensen and Gøtzsche, 2010), partly revolve around length of follow-up as the benefits of screening persist over many years.

The lack of clarity regarding treatment options, and the increasing trend in the US to treat DCIS with bilateral mastectomy (Tuttle et al, 2009) also raises the spectre of overtreatment. The very earliest study of untreated DCIS was confined to a small number of low-grade cases, with a $39 \%$ (11 of 28) conversion to invasive disease after a median follow-up of 31 years (Sanders et al, 2005), but an overview in 2006 (Mokbel and Cutuli, 2006), reported rates varying from 14 to $75 \%$. Recurrence rates in both the randomised trials and observational studies are very variable. The overview by Mokbel (Mokbel and Cutuli, 2006) reports recurrence rates of $1.5 \%$ after mastectomy, $33-56 \%$ after conservation alone and 3 to $19 \%$ after conservation with radiotherapy, but the studies contributing to these figures have a wide range of follow-up (median follow-up 57 to 120 months).

Whereas there is uniform agreement regarding the importance of age and a clear margin (even if there is no agreement as to the definition of 'clear') for reducing recurrence, the literature is divided as to whether biological factors (size and grade) are as predictive (Welch et al, 2008; Virnig et al, 2010). Solin found that grade was important after 5 years but not at 10 years (Solin et al, 1996). Harris' accompanying editorial concludes that longer-term follow-up is important particularly, if looking for specific types of 
DCIS where minimal treatment could be considered (Harris and Morrow, 2009).

Estimates of the rate of recurrence, as invasive disease, range from between one-third and one-half, and there is minimal data on features predicting invasive as opposed to noninvasive recurrence. An invasive recurrence is clearly predictive for the development of metastatic disease and subsequent mortality (Mokbel and Cutuli, 2006).

We present a minimum of 11-years follow-up (median 183 months, range 133 months to 259 months) of a cohort of screendetected DCIS cases diagnosed between April 1988 and March 1999; all graded retrospectively, by one expert pathologist.

\section{MATERIALS AND METHODS}

The study population consisted of 840 cases of noninvasive breast cancer without evidence of invasive disease recorded between 1 March 1988 and 31 March 1999 on the National Breast Screening Computer System in 10 West Midlands breast screening services. 674 cases were DCIS alone, and 144 were DCIS with microinvasion or possible micro-invasion. Of the remaining cases, 15 were lobular carcinoma in situ and 7 were Paget's disease of the breast. All cases were allocated a study ID number, and their demographic details were entered on to the study database. Diagnosis, treatment and follow-up data, recorded for these cases on the cancer registration database and in-patient case notes held by the West Midlands Cancer Intelligence Unit (WMCIU), were added to the study database. At this stage, 71 cases were excluded ( 35 were not DCIS, 10 were bilateral, 15 were second primary breast cancers and 11 were recurrences) (Figure 1).

Data not available at the WMCIU were sought in and abstracted from the original case notes stored at the treating hospitals, using a specialist data collection proforma. A pathological slide review was undertaken by a consultant pathologist to provide consistent information on diagnostic characteristics. The slides had patient identifiers obscured to ensure anonymity. Furthermore, to deal

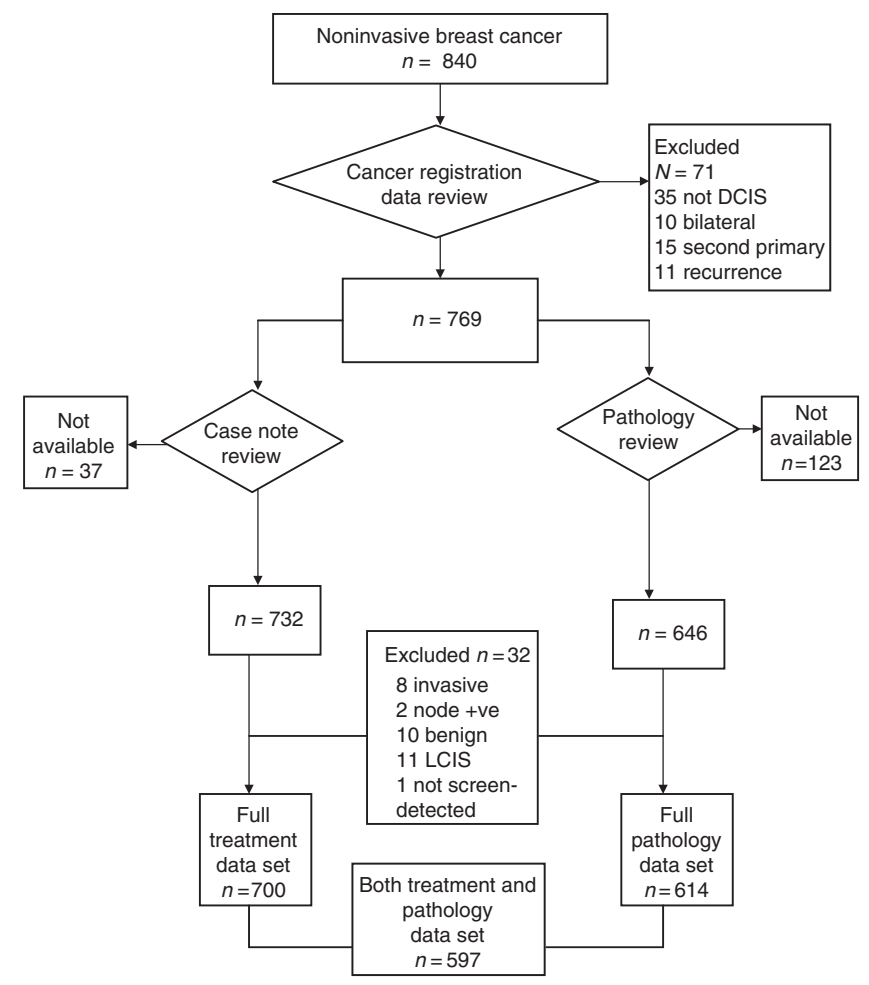

Figure I STARD diagram showing case numbers. with the potential problem of the review pathology being significantly different from the original pathology, a comprehensive double-blinding process (under the terms of Multi-centre Research Ethics Committee approval) was used throughout the project. Once the data collection process was complete, the doubleblinding process was applied to the completed database. It was then possible to link, on a case-by-case basis, data from the treatment and follow-up review with data derived from the slide review, while ensuring that it was impossible to identify individuals. 123 cases were excluded from the pathology review and 37 from the case note review, owing to problems with slide and case note availability. A further 32 cases were excluded following pathology and case note review, because they were found not to be DCIS ( 8 had invasive disease, 2 cases were node positive, although no invasive disease was found in the original resection, 10 had benign disease and 11 were LCIS cases), and one because it was detected symptomatically rather than through screening. After the exclusions, 700 cases were identified with a full-treatment dataset, 614 cases were subjected to a full pathology review, and 597 cases had both pathology and treatment datasets (Figure 1).

To maximise the number of 'known' data items, information relating to margin status, nuclear grade and tumour size was obtained from the original pathology reports and from the pathology review. For margin status, the margin size was taken from the original report, if the actual distance was stated. If this was not the case, the distance measured in the pathology review was used. If the actual distance was not available from either source, a descriptive margin status (e.g. 'clear') was used. If no information on margin status was available from either source, the margin status was recorded as unknown. For tumour size, the size from the pathology review was used in preference to that in the original pathology report, to maximise consistency. If tumour size was not available from either source, the size was recorded as unknown. The nuclear grade was also preferentially taken from the pathology review data. If nuclear grade was not available from either source, the nuclear grade was recorded as unknown.

Cases were followed up to 30 June 2010 via the WMCIU. Local and regional recurrences and distant metastatic events, along with date of death and underlying cause of death, were identified from the WMCIU's cancer registration database. Invasive recurrences, after a first primary diagnosed as DCIS, are registered as multiple primary cancers in their own right.

Statistical analyses were performed using SPSS (Version 13.0, SPSS Inc., Chicago, IL, USA), Statistica (Statsoft Ltd, Tulsa, OK, USA) and Microsoft Excel (Microsoft Corp., Seattle, WA, USA). $\chi^{2}$ tests, with Bonferonni corrections for multiple testing, where appropriate, compared use of adjuvant therapy between patients treated with conservative surgery and those treated by mastectomy, and between patients with clear and with involved excision margins. Numbers of recurrences were compared using $\chi^{2}$ tests between patients with different treatments, margins, grades and Van Nuys Prognostic Index (VNPI). Recurrence rates were compared for these groups using a Kaplan-Meier chart and a log-rank test, and times to recurrence using a $t$-test. All events including censored cases were included in the Kaplan-Meier plots and analysis.

Multivariate Cox regression was utilised to determine significant factors related to recurrence.

\section{RESULTS}

\section{Pathological characteristics}

As shown in Table 1, for the 700 cases with a full treatment data set, micro-calcification was present in a high proportion of cases $(74.3 \%)$; moderate or severe necrosis ( $>5 \%$ ) was visible in $51.3 \%$ of cases; and $49.1 \%$ of cases were of high nuclear grade. Tumour 
size was recorded for $57.9 \%$ of cases. In the majority of cases with known tumour size $(65.4 \%)$, the DCIS was $15 \mathrm{~mm}$ or less in diameter; in only $1 \%$ of cases was the diameter greater than $40 \mathrm{~mm}$. An exact distance to the nearest tumour margin was available for $42.6 \%$ of cases and some description of margin status was available for $90.7 \%$.

The VNPI calculation depends on lesion size, margin width, and a classification based on nuclear grade and necrosis (Silverstein et al, 1996). At least one of the required data items was missing from 347 cases, so a VNPI score could only be calculated for 353 cases $(50.4 \%)$. Of these, $81(22.9 \%)$ were in the best prognostic groups (VNPI score 3 or 4); 255 (72.2\%) had a VNPI score of 5, 6 or 7; and $17(4.8 \%)$ had a VNPI score of 8 . No lesions had a VNPI score of 9 .

\section{Treatment data}

Table 2 summarises the surgical and adjuvant treatment received. 449 cases $(64.2 \%)$ had breast-conserving surgery as their final therapeutic operation and $251(35.8 \%)$ had a mastectomy. Overall, a significantly higher proportion of cases treated with breastconserving surgery (289 cases, $64.3 \%$ ) received adjuvant therapy compared with cases treated with mastectomy (108 cases, $42.8 \%$; $P<0.00001) .143$ cases treated with breast-conserving surgery $(31.7 \%)$ received adjuvant radiotherapy compared with only four cases treated with mastectomy $(1.6 \% ; P<0.00001)$. Patients treated with mastectomy were more likely to receive hormone therapy as their only adjuvant treatment $(104,41.6 \%)$ than those treated with conservative surgery $(144,32.1 \%)$. This tended towards significance but was not the case when corrections for multiple testing were applied. However, patients treated with breast-conserving surgery were more likely to receive hormone therapy $(252,56.0 \%)$ than patients who had a mastectomy $(106,42.4 \% ; P<0.0005)$. Of the 358 patients receiving hormone therapy, 10 (2.8\%) reported stopping treatment within 2 months.

\section{Follow-up}

Table 3 shows the number and type of first ipsilateral and metastatic events, and number and cause of deaths up to 30 June 2010 , and splits these according to the initial grade of the DCIS. After a median follow-up of 183 months (range 133 to 259 months), 102 (14.6\%) first-isolated local recurrences in the ipsilateral breast and 4 local recurrences with contra-lateral disease $106(15.1 \%)$ were identified. $49(48 \%)$ of the isolated local recurrences were invasive and eventually 54 (50.1\%) of the 106 developed invasive disease in the ipsilateral breast. A further seven women presented with metastases.

37 contra-lateral events were recorded; 30 (81\%) were invasive. Life tables (Table 4) constructed to examine ipsilateral and first metastatic events show that, at 60 months, the cumulative proportion developing recurrence was $8.6 \% \quad(\mathrm{HR}=0.0004$, $n=613)$. At 180 months, the recurrence rate almost doubled to $16 \%$ (HR $0.0016 n=255$ ). Age, size, grade, margin, and necrosis

Table I Pathology characteristics of the 700 cases with treatment data using data from the pathology review and original pathology reports for micro-calcification, necrosis, nuclear grade, tumour size and margin

\begin{tabular}{|c|c|c|c|c|c|c|c|c|c|c|c|c|c|}
\hline \multirow[b]{2}{*}{ Characteristic } & & \multicolumn{2}{|c|}{ All cases } & \multicolumn{2}{|c|}{$\begin{array}{l}\text { VNPI } \\
\text { groups }\end{array}$} & \multicolumn{2}{|c|}{$\begin{array}{l}\text { Conservation } \\
\text { surgery (CS) }\end{array}$} & \multicolumn{2}{|c|}{$\begin{array}{l}\text { VNPI } \\
\text { groups }\end{array}$} & \multicolumn{2}{|c|}{$\begin{array}{l}\text { Mastectomy } \\
\text { (MX) }\end{array}$} & \multicolumn{2}{|c|}{$\begin{array}{l}\text { VNPI } \\
\text { groups }\end{array}$} \\
\hline & & No. & $\begin{array}{c}\% \text { of all } \\
\text { cases }\end{array}$ & No. & $\%$ & No. & $\begin{array}{l}\% \text { of all } \\
\text { CS cases }\end{array}$ & No. & $\%$ & No. & $\begin{array}{c}\% \text { of all } \\
\text { MX cases }\end{array}$ & No. & $\%$ \\
\hline Microcalcification & $\begin{array}{l}\text { Present } \\
\text { Not present } \\
\text { Unknown }\end{array}$ & $\begin{array}{r}520 \\
76 \\
104\end{array}$ & $\begin{array}{l}74.29 \\
10.86 \\
14.86\end{array}$ & & & $\begin{array}{r}324 \\
51 \\
74\end{array}$ & $\begin{array}{l}72.16 \\
11.36 \\
16.48\end{array}$ & & & $\begin{array}{r}196 \\
25 \\
30\end{array}$ & $\begin{array}{r}78.09 \\
9.96 \\
11.95\end{array}$ & & \\
\hline Necrosis & $\begin{array}{l}\text { None } \\
<5 \% \\
5-50 \% \\
>50 \% \\
\text { Unknown }\end{array}$ & $\begin{array}{r}165 \\
63 \\
188 \\
171 \\
113\end{array}$ & $\begin{array}{r}23.57 \\
9.00 \\
26.86 \\
24.43 \\
16.14\end{array}$ & & & $\begin{array}{r}108 \\
37 \\
119 \\
105 \\
80\end{array}$ & $\begin{array}{r}24.05 \\
8.24 \\
26.50 \\
23.39 \\
17.82\end{array}$ & & & $\begin{array}{l}57 \\
26 \\
69 \\
66 \\
33\end{array}$ & $\begin{array}{l}22.71 \\
10.36 \\
27.49 \\
26.29 \\
13.15\end{array}$ & & \\
\hline Nuclear grade & $\begin{array}{l}\text { Low } \\
\text { Intermediate } \\
\text { High } \\
\text { Unknown } \\
\text { Non- } \\
\text { high + necrosis } \\
\text { Non-high - necrosis }\end{array}$ & $\begin{array}{r}68 \\
174 \\
344 \\
114 \\
124 \\
118\end{array}$ & $\begin{array}{r}9.71 \\
24.86 \\
49.14 \\
16.29 \\
17.71 \\
16.86\end{array}$ & $\begin{array}{l}344 \\
114 \\
124 \\
118\end{array}$ & $\begin{array}{l}49.14 \\
16.29 \\
17.71 \\
16.86\end{array}$ & $\begin{array}{r}47 \\
112 \\
210 \\
80 \\
78 \\
81\end{array}$ & $\begin{array}{l}10.47 \\
24.94 \\
46.77 \\
17.82 \\
17.37 \\
18.04\end{array}$ & $\begin{array}{r}210 \\
80 \\
78 \\
\\
81\end{array}$ & $\begin{array}{l}46.77 \\
17.82 \\
17.37 \\
18.04\end{array}$ & $\begin{array}{r}21 \\
62 \\
134 \\
34 \\
46 \\
\\
37\end{array}$ & $\begin{array}{r}8.37 \\
24.70 \\
53.39 \\
13.55 \\
18.33 \\
14.74\end{array}$ & $\begin{array}{r}134 \\
34 \\
46 \\
\\
37\end{array}$ & $\begin{array}{l}53.39 \\
13.55 \\
18.33 \\
14.74\end{array}$ \\
\hline Original tumour size & $\begin{array}{l}<5 \mathrm{~mm} \\
5-10 \mathrm{~mm} \\
11-15 \mathrm{~mm} \\
16-20 \mathrm{~mm} \\
21-30 \mathrm{~mm} \\
31-40 \mathrm{~mm} \\
>40 \mathrm{~mm} \\
\text { Unknown }\end{array}$ & $\begin{array}{r}31 \\
110 \\
124 \\
91 \\
41 \\
4 \\
4 \\
295\end{array}$ & $\begin{array}{r}4.43 \\
15.7 \mid \\
17.7 \mid \\
13.00 \\
5.86 \\
0.57 \\
0.57 \\
42.14\end{array}$ & $\begin{array}{r}265 \\
136 \\
4 \\
295\end{array}$ & $\begin{array}{r}37.86 \\
19.43 \\
0.57 \\
42.14\end{array}$ & $\begin{array}{r}23 \\
97 \\
88 \\
65 \\
18 \\
2 \\
3 \\
153\end{array}$ & $\begin{array}{r}5.12 \\
21.60 \\
19.60 \\
14.48 \\
4.01 \\
0.45 \\
0.67 \\
34.08\end{array}$ & $\begin{array}{r}208 \\
85 \\
3 \\
153\end{array}$ & $\begin{array}{r}46.33 \\
18.93 \\
0.67 \\
34.08\end{array}$ & $\begin{array}{r}8 \\
13 \\
36 \\
26 \\
23 \\
2 \\
1 \\
142\end{array}$ & $\begin{array}{r}3.19 \\
5.18 \\
14.34 \\
10.36 \\
9.16 \\
0.80 \\
0.40 \\
56.57\end{array}$ & $\begin{array}{r}51 \\
1 \\
142\end{array}$ & $\begin{array}{r}22.71 \\
20.32 \\
0.40 \\
56.57\end{array}$ \\
\hline Margin status & $\begin{array}{l}<1.0 \mathrm{~mm} \\
1.00-4.99 \mathrm{~mm} \\
5.00-9.99 \mathrm{~mm} \\
\geqslant 10.00 \mathrm{~mm} \\
\text { Clear } \\
\text { Probably clear } \\
\text { Probably involved } \\
\text { Involved } \\
\text { Unknown }\end{array}$ & $\begin{array}{r}18 \\
156 \\
67 \\
57 \\
205 \\
43 \\
32 \\
57 \\
65\end{array}$ & $\begin{array}{r}2.57 \\
22.29 \\
9.57 \\
8.14 \\
29.29 \\
6.14 \\
4.57 \\
8.14 \\
9.29\end{array}$ & $\begin{array}{r}18 \\
223 \\
57\end{array}$ & $\begin{array}{r}2.57 \\
31.86 \\
8.14\end{array}$ & $\begin{array}{r}16 \\
120 \\
45 \\
24 \\
111 \\
24 \\
26 \\
51 \\
32\end{array}$ & $\begin{array}{r}3.56 \\
26.73 \\
10.02 \\
5.35 \\
24.72 \\
5.35 \\
5.79 \\
11.36 \\
7.13\end{array}$ & $\begin{array}{r}16 \\
165 \\
24\end{array}$ & $\begin{array}{r}3.56 \\
36.75 \\
5.35\end{array}$ & $\begin{array}{r}2 \\
36 \\
23 \\
33 \\
94 \\
19 \\
6 \\
6 \\
33\end{array}$ & $\begin{array}{r}0.80 \\
14.34 \\
9.16 \\
13.15 \\
37.45 \\
7.57 \\
2.39 \\
2.39 \\
13.15\end{array}$ & $\begin{array}{r}2 \\
59 \\
24\end{array}$ & $\begin{array}{r}0.80 \\
23.51 \\
9.56\end{array}$ \\
\hline
\end{tabular}


Table 2 Adjuvant therapy for 700 cases with full treatment data

\begin{tabular}{|c|c|c|c|c|c|c|c|}
\hline \multirow[b]{2}{*}{ Treatment } & \multicolumn{2}{|c|}{ Conservation surgery } & \multicolumn{2}{|c|}{ Mastectomy } & \multicolumn{2}{|c|}{ All cases } & \multirow[b]{2}{*}{$P$} \\
\hline & No. & $\%$ & No. & $\%$ & No. & $\%$ & \\
\hline Surgery type & 449 & 64.14 & 251 & 35.86 & 700 & 100.00 & \\
\hline No radiotherapy & 298 & 66.37 & 243 & 96.81 & 541 & 77.29 & $<0.00001$ \\
\hline Any radiotherapy ${ }^{a}$ & 143 & 31.85 & 4 & 1.59 & 147 & 21.00 & $<0.00001$ \\
\hline Unknown Radiotherapy & 8 & 1.78 & 4 & 1.59 & 12 & 1.71 & \\
\hline Radiotherapy only & 36 & 8.02 & 2 & 0.80 & 38 & 5.43 & $<0.0001$ \\
\hline No hormone therapy & 189 & 42.09 & 138 & 54.98 & 327 & 46.71 & $<0.01^{\mathrm{b}}$ \\
\hline Any hormone therapy ${ }^{c}$ & 252 & 56.12 & 106 & 42.23 & 358 & 51.14 & $<0.0005$ \\
\hline Unknown hormone therapy & 8 & 1.78 & 7 & 2.79 & 15 & 2.14 & \\
\hline Hormone therapy only & 144 & 32.07 & 104 & 41.43 & 248 & 35.43 & $<0.05^{b}$ \\
\hline Radiotherapy + hormone therapy & 106 & 23.61 & 2 & 0.80 & 108 & 15.43 & $<0.00001$ \\
\hline Other combinations & 10 & 2.23 & 7 & 2.79 & 17 & 2.43 & \\
\hline Any known adjuvant therapy & 286 & 63.70 & 108 & 43.03 & 394 & 56.29 & $<0.00001$ \\
\hline No adjuvant therapy & 153 & 34.08 & 136 & 54.18 & 289 & 41.29 & $<0.00001$ \\
\hline
\end{tabular}

All cases of hormone therapy given are included in this table, no matter how long the hormone therapy was continued.

a'Any radiotherapy' includes patients who had radiotherapy alone or radiotherapy + hormone therapy or radiotherapy + unknown hormone therapy.

${ }^{b} \mathrm{NS}$ when corrected for multiple testing.

cAny hormone therapy' includes patients who had hormone therapy alone or hormone therapy + radiotherapy or hormone therapy + unknown radiotherapy.

Table 3 Number of first non-contralateral events and breast cancer deaths up to and including June 2010 diagnosed in the first 10 years of West Midlands Breast Screening programme between Apr 1988 and March 1998

\begin{tabular}{|c|c|c|c|c|c|c|c|c|c|c|c|c|c|c|c|c|c|c|}
\hline \multirow[b]{2}{*}{$\begin{array}{l}\text { Initial } \\
\text { grade }\end{array}$} & \multicolumn{7}{|c|}{ First 'non-contra-lateral event' } & \multicolumn{11}{|c|}{ Number and type of deaths } \\
\hline & $\begin{array}{l}\text { No. } \\
\text { of } \\
\text { cases }\end{array}$ & $\begin{array}{l}\text { No. of } \\
\text { 'non- } \\
\text { contra- } \\
\text { lateral } \\
\text { events' }\end{array}$ & $\%$ & $\begin{array}{c}\text { Type } \\
\text { of } \\
\text { event }\end{array}$ & $\begin{array}{l}\text { No. of } \\
\text { events }\end{array}$ & $\begin{array}{c}\% \\
\text { with } \\
\text { event }\end{array}$ & $\begin{array}{c}\% \text { of } \\
\text { grade } \\
\text { category } \\
\text { overall }\end{array}$ & $\begin{array}{l}\text { Deaths } \\
\text { from } \\
\text { breast } \\
\text { cancer } \\
\text { after } \\
\text { one } \\
\text { 'non } \\
\text { contra- } \\
\text { lateral } \\
\text { event' }\end{array}$ & $\begin{array}{c}\text { Deaths } \\
\text { from } \\
\text { breast } \\
\text { cancer } \\
\text { after one } \\
\text { non } \\
\text { contra- } \\
\text { lateral } \\
\text { event }+ \text { a } \\
\text { further } \\
\text { event }\end{array}$ & $\begin{array}{c}\text { Deaths } \\
\text { from } \\
\text { breast } \\
\text { cancer } \\
\text { after } \\
\text { bi- } \\
\begin{array}{c}\text { lateral } \\
\text { further } \\
\text { event }\end{array}\end{array}$ & $\begin{array}{c}\text { Deaths } \\
\text { from } \\
\text { breast } \\
\text { cancer } \\
\text { after } \\
\text { contra- } \\
\text { lateral } \\
\text { further } \\
\text { event }\end{array}$ & $\begin{array}{l}\text { Total } \\
\text { deaths } \\
\text { from } \\
\text { breast } \\
\text { cancer }\end{array}$ & $\begin{array}{c}\text { Mortality } \\
(\%)\end{array}$ & $\begin{array}{c}\text { Number } \\
\text { of non- } \\
\text { cancerl } \\
\text { other } \\
\text { cancerl } \\
\text { other } \\
\text { breast } \\
\text { primary } \\
\text { deaths }\end{array}$ & $\begin{array}{c}\text { Mortality } \\
(\%)\end{array}$ & $\begin{array}{c}\text { Number } \\
\text { of } \\
\text { unknown } \\
\text { cause of } \\
\text { death }\end{array}$ & $\begin{array}{l}\text { Deaths } \\
\text { from all } \\
\text { causes }\end{array}$ & $\begin{array}{c}\text { Mortality } \\
\text { (\%) }\end{array}$ \\
\hline \multirow[t]{4}{*}{ Low } & 68 & 10 & $14.7 \%$ & $\begin{array}{l}\text { DCIS/ } \\
\text { Pagets }\end{array}$ & 4 & 40.00 & 5.88 & 0 & 0 & 0 & & 0 & & & & & & \\
\hline & & & & Invasive & 6 & 60.00 & 8.82 & 0 & 0 & 0 & & 0 & & & & & & \\
\hline & & & & Mets & 0 & 0.00 & 0.00 & 0 & 0 & 0 & & 0 & & & & & & \\
\hline & & & & all & 10 & 100.00 & |4.7| & 0 & 0 & 0 & 0 & 0 & 0.00 & 8 & 11.76 & 0 & 8 & 11.76 \\
\hline \multirow[t]{4}{*}{$\begin{array}{l}\text { Inter- } \\
\text { mediate }\end{array}$} & 174 & 28 & $16.1 \%$ & $\begin{array}{l}\text { DCIS/ } \\
\text { Pagets }\end{array}$ & 14 & 50.00 & 8.05 & 0 & 0 & 0 & & 0 & & & & & & \\
\hline & & & & Invasive & 9 & 32.14 & 5.17 & I & 1 & 1 & & 3 & & & & & & \\
\hline & & & & Mets & 5 & 17.86 & 2.87 & 3 & 0 & 0 & & 3 & & & & & & \\
\hline & & & & all & 28 & 100.00 & 16.09 & 4 & 1 & I & 0 & 6 & 3.45 & 16 & 9.20 & 1 & 23 & 13.22 \\
\hline \multirow[t]{4}{*}{ High } & 344 & 53 & $15.4 \%$ & $\begin{array}{l}\text { DCIS/ } \\
\text { Pagets }\end{array}$ & 29 & 54.72 & 8.43 & 0 & 1 & 0 & & 1 & & & & & & \\
\hline & & & & Invasive & 22 & 41.51 & 6.40 & I & 4 & I & & 6 & & & & & & \\
\hline & & & & Mets & 2 & 3.77 & 0.58 & I & 1 & 0 & & 2 & & & & & & \\
\hline & & & & all & 53 & 100.00 & $|5.4|$ & 2 & 6 & । & 1 & 10 & 2.91 & 49 & 14.24 & I & 60 & 17.44 \\
\hline \multirow[t]{4}{*}{ Unknown } & 114 & 18 & $15.8 \%$ & $\begin{array}{l}\text { DCIS/ } \\
\text { Pagets }\end{array}$ & 6 & 33.33 & 5.26 & 0 & 0 & 0 & & 0 & & & & & & \\
\hline & & & & Invasive & 12 & 66.67 & 10.53 & 2 & 6 & 0 & & 8 & & & & & & \\
\hline & & & & Mets & 0 & 0.00 & 0.00 & 0 & 0 & 0 & & 0 & & & & & & \\
\hline & & & & & 18 & 100.00 & 15.79 & 2 & 6 & 0 & I & 9 & 7.89 & 8 & 7.02 & 0 & 17 & $14.9 \mid$ \\
\hline \multirow[t]{4}{*}{ Total } & 700 & 109 & $15.6 \%$ & $\begin{array}{l}\text { DCIS/ } \\
\text { Pagets }\end{array}$ & 53 & 48.62 & 7.57 & 0 & 1 & 0 & & 1 & & & & & & \\
\hline & & & & Invasive & 49 & 44.95 & 7.00 & 4 & 11 & 2 & & 17 & & & & & & \\
\hline & & & & Mets & 7 & 6.42 & 1.00 & 4 & 1 & 0 & & 5 & & & & & & \\
\hline & & & & all & 109 & 100.00 & 15.57 & 8 & 13 & 2 & 2 & 25 & 3.57 & 81 & 11.57 & 2 & 108 & 15.43 \\
\hline
\end{tabular}

were not predictive for recurrence either singly or in multi-variant analysis.

For women treated with breast-conserving surgery at 60 months, the cumulative proportion-developing reoccurrence was $11.8 \%$ (HR $0.0002, n=379$ ); by 180 months, this rose to $21.5 \%$ (HR 0.002 , $n=166$ ). Tumour size is the only single predictor. For those treated by mastectomy, the cumulative proportion developing recurrence at 60 months was $3.1 \%$ (HR $0.0007, n=234$ ); by 180 months, this was $8 \%$ (HR $0.001, n=89$ ). Tumour size and necrosis tend to significance but less than for breast-conserving surgery $(P=0.08)$.

\section{Time to recurrence}

Of those with known original DCIS grade, median time to first local non contra-lateral event was 15 months for noninvasive recurrence, regardless of original DCIS grade. Overall, the median time to invasive recurrence was 60 months. This was grade dependent. Median time to invasive recurrence from initially highgrade DCIS was 76 months, and from low-/intermediate-grade DCIS was 131 months (Figure 2). Median time for metastasis, as first event, was 82 months (range 15 to 188 months). 
The histological grades of the invasive recurrences, which were not centrally reviewed, are documented in Table 5. The numbers are small but no high-grade invasive disease arose from low-grade

Table 4 Life tables for ipsilateral invasive recurrences up to 30 June 2010 from DCIS diagnosed in the West Midlands screening programme between April 1988 and March 1998

\begin{tabular}{llccc}
\hline $\begin{array}{l}\text { Time } \\
\text { (months) }\end{array}$ & $\begin{array}{l}\text { Number } \\
\text { entering }\end{array}$ & $\begin{array}{c}\text { Cumulative } \\
\text { event rate } \\
(\%)\end{array}$ & $\begin{array}{c}\text { Hazard } \\
\text { rates }\end{array}$ & $\begin{array}{c}\text { No } \\
\text { recurrence }\end{array}$
\end{tabular}

\begin{tabular}{|c|c|c|c|c|}
\hline \multicolumn{5}{|c|}{ Breast-conserving surgery } \\
\hline 0 & 448 & & & \\
\hline 60 & 379 & 11.8 & 0.0002 & $88.2 \pm 1.5$ \\
\hline 120 & 332 & 17.1 & 0.0007 & $82.9 \pm 1.8$ \\
\hline 180 & 166 & 21.5 & 0.002 & $78.5 \pm 2.0$ \\
\hline
\end{tabular}

\begin{tabular}{cccll}
$\begin{array}{c}\text { Mastectomy } \\
0\end{array}$ & 250 & & & \\
60 & 234 & 3.1 & 0.0007 & $96.9 \pm 1.1$ \\
120 & 218 & 5.6 & 0.0004 & $94.4 \pm 1.4$ \\
180 & 89 & 8 & 0.01 & $92.0 \pm 1.8$ \\
& & & & \\
Total & & & & \\
0 & 700 & & & \\
60 & 613 & 8.6 & 0.0004 & $91.4 \pm 1.3$ \\
120 & 550 & 12.6 & 0.0006 & $87.4 \pm 1.6$ \\
180 & 255 & 16 & 0.0016 & $84.0 \pm 1.9$ \\
\hline
\end{tabular}

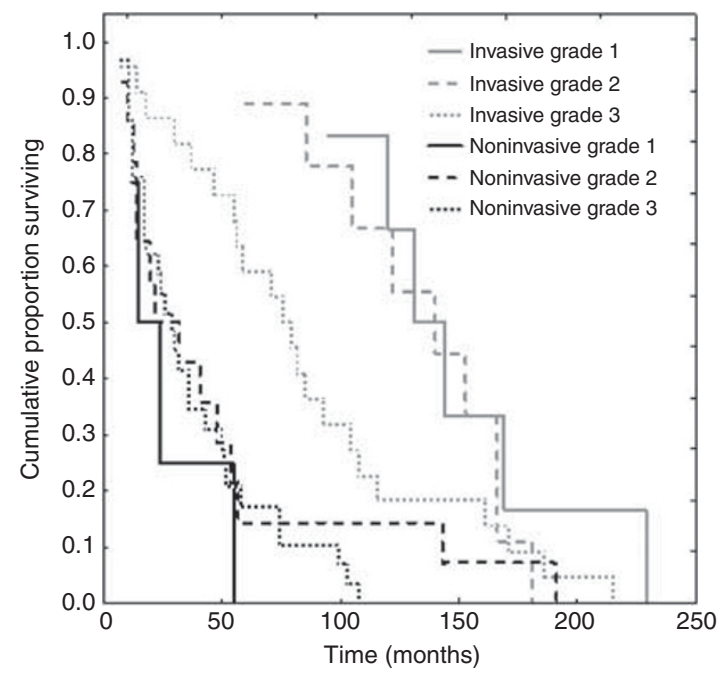

Figure 2 Time to first recurrence (invasive and noninvasive) according to original grade.
DCIS, and, of the 28 invasive recurrences (of known grade) arising from intermediate or high-grade DCIS, only one was grade 1.

In total, $108(15.4 \%)$ women died before 30 June 2010. Twenty Five $(3.6 \%)$ died from breast cancer (Table 5). There were no breast cancer deaths in the low-grade DCIS group, and similar rates in the intermediate $(3.4 \%)$ and the high (3.2\%)-grade groups. The majority of the deaths $(82 / 108 ; 76 \%)$ were unrelated to the women's breast cancer diagnosis. Of the 54 cases with an ipsilateral invasive recurrence, 18 had died of breast cancer by the census date. Of the cases who died of breast cancer, $70 \%$ had involved, or unknown margin status compared with $35.3 \%$ of the cases, who were still alive.

\section{DISCUSSION}

At $15.1 \%$ (including those with bilateral disease) (cumulatively $21.5 \%$ after breast-conserving surgery and $8 \%$ post mastectomy), the overall first local recurrence rates from this observational cohort are well within the ranges quoted in the two recent overviews (Mokbel and Cutuli, 2006; Virnig et al, 2010). What is important to note is that events continue to occur throughout the 259 months of follow-up. For women treated by breast-conserving surgery, the rate almost doubles between 60 and 180 months and it more than doubles over the same time period for women treated by mastectomy, indicating that event rates do not drop or stabilise with time. This suggests that the 'cure' model (Lambert et al, 2007) that has been applied to invasive cancers is not applicable to DCIS and that standard short term follow-up will miss a significant number of events.

Our finding that, after 183 months, the absolute ipsilateral event rates are not DCIS grade dependent is in keeping with Solin et al (2001) (median follow-up 9.4 yr range 1-19 yrs) but contradicts Bijker et al (2001) (5.4 yrs), Nakamura et al (2002) (105 months), Kerlikowske et al (2003) (77.9 months) and Pinder et al (2010) (5.7 yrs). Bijker et al (2001) and Kerlikowske et al (2003) did not find that the type of recurrence (i.e. invasive or noninvasive was related to the original DCIS grade, but Bijker et al (2001) does report that the development of metastatic disease is strongly associated with high-grade DCIS, as in our series.

Our long period of follow-up has enabled us to look at type and timing of first recurrence and relate this to original DCIS grade. Noninvasive recurrence occurs early and this is not dependent on the original grade of the DCIS. In contrast, invasive local recurrence not only presents later, but the median time is DCIS grade dependent too.

High-grade DCIS starts to recur early (under 6 months) and continues throughout the period of follow-up, with a median time of 76 months, whereas low/intermediate grade DCIS only starts to recur after 60 months (most of the noninvasive events have occurred by this point), with a median time of 131 months. Only Solin et al (2001) have reported time to local

Table 5 Histological grade of ipsilateral invasive recurrences up to and including December 2010 from DCIS diagnosed in the West Midlands screening programme between April 1988 and March 1998 by initial DCIS grade

\begin{tabular}{|c|c|c|c|c|c|c|c|c|c|c|c|}
\hline \multirow{3}{*}{$\begin{array}{l}\text { Original DCIS } \\
\text { grade }\end{array}$} & \multicolumn{11}{|c|}{$\begin{array}{l}\text { Ipsilateral invasive recurrence (all events) } \\
\text { Grade }\end{array}$} \\
\hline & \multirow[b]{2}{*}{ No. } & \multicolumn{2}{|c|}{ I } & \multicolumn{2}{|c|}{2} & \multicolumn{2}{|c|}{3} & \multicolumn{2}{|c|}{ N/K } & \multirow[b]{2}{*}{ Total } & \multirow{2}{*}{$\begin{array}{c}\text { Breast cancer } \\
\text { deaths }\end{array}$} \\
\hline & & No. & $\%$ & No. & $\%$ & No. & $\%$ & No. & $\%$ & & \\
\hline Low & 68 & 2 & $33.3 \%$ & 2 & $33.3 \%$ & 0 & $0.0 \%$ & 2 & $33.3 \%$ & 6 & 0 \\
\hline Intermediate & 174 & 0 & $0.0 \%$ & 4 & $36.4 \%$ & 5 & $45.5 \%$ & 2 & | $8.2 \%$ & 11 & 3 \\
\hline High & 344 & 1 & $4.0 \%$ & 10 & $40.0 \%$ & 8 & $32.0 \%$ & 6 & $24.0 \%$ & 25 & 7 \\
\hline Not known & 114 & 0 & $0.0 \%$ & 6 & $50.0 \%$ & 5 & $41.7 \%$ & I & $8.3 \%$ & 12 & 8 \\
\hline Total & 700 & 3 & $5.6 \%$ & 22 & $40.7 \%$ & 18 & $33.3 \%$ & | | & $20.4 \%$ & 54 & 18 \\
\hline
\end{tabular}


recurrence, and he found no statistical difference between median time to invasive recurrence ( 4.8 years) and noninvasive recurrence (5.1 years).

As expected there have been few deaths in our DCIS cohort, but, in line with Lee et al (2006) and Wapnir et al (2011), these deaths have been in those women who either presented with metastatic disease as their first event, or had an ipsilateral invasive recurrence as their first or subsequent events. To date we have not identified any high-grade recurrences, metastatic events or deaths from our cohort of 68 low-grade DCIS patients. This is in keeping with Nakamura et al (2002) who also showed lower local recurrence rates and no deaths in his low-grade population. Of those patients with an ipsilateral invasive recurrence who had died by the study census date, $70 \%$ had involved or unknown margin status compared with only $35.3 \%$ of those who were still alive.

The main limitation of this study is that it is a cohort of women over 50 years of age with mixed, uncontrolled treatment. As in other studies from the period, we have limited information on margin status that cannot be fully resolved by pathological review (Bijker et al, 2001; Pinder et al, 2010) reflecting the more variable pathological practice encountered earlier in the UK screening programme. The grade distribution (49\% high grade and 10\% low grade) is clearly different to the EORTC randomised trial (40\% poorly and 32\% well-differentiated) (Bijker et al, 2001), which had higher rates of low-grade/well-differentiated DCIS (however, this trial recruited both clinically and mammographically detected DCIS treated by conservation surgery) and to the UKCCCR/ANZ (75\% high and $7 \%$ low grade) (Lee et al, 2006). The distribution is not that different to the current UK data from the NHSBSP/ABS 2010 audit of screen-detected cancers (58\% high grade and $10 \%$ low grade). An advantage of our study is that central pathological review by a single expert does provide uniformity and excludes cases that have been under or over reported by local pathologists (Nakamura et al, 2002; Pinder et al, 2010).

The long follow-up in the present study supports the concept that DCIS is a long-term risk factor that will bias studies of overdiagnosis with short-term follow-up. The differential effect of DCIS grade on type of recurrence and time to invasive recurrence supports the model suggesting that breast cancer evolves along two distinct molecular genetic pathways (Meijnen et al, 2008; Bombonati and Sgroi, 2011), particularly as we have not identified any high-grade recurrences, metastatic events or deaths from our cohort of low-grade DCIS.

It would be nice to try and use our data as a basis to calculate or recalculate overdiagnosis rates from DCIS (Jørgensen and Gøtzsche, 2009), but all of these women received surgical treatment and many had additional radiotherapy that is not the same as leaving them untreated. The low event rates over a prolonged time reinforce the rationale for aggressive treatment of younger women who have higher event rates (Joslyn, 2006) compared with older women. Whether the same treatment policies should be used for older women who could well outlive their disease, or women with low-grade DCIS, is open for debate. We are aware of discussion about a randomised trial comparing conventional surgical treatment with active radiological monitoring and we consider our data support this approach.

\section{REFERENCES}

Bijker N, Peterse JL, Duchateau L, Julien JP, Fentiman IS, Duval C, Di Palma S, Simony-Lafontaine J, de Mascarel I, van de Vijver MJ (2001) Risk factors for recurrence and metastasis after breast-conserving therapy for ductal carcinoma-in-situ: analysis of European Organisation for Research and Treatment of Cancer Trial 10853. J Clin Oncol 19: 2263-2271

Bombonati A, Sgroi DC (2011) The molecular pathology of breast cancer progression. J Pathol 223: 307-317

Duffy SW, Tabar L, Olsen AH, Vitak B, Allgood PC, Chen THH, Yen AMF, Smith RA (2010) Absolute numbers of lives saved and over diagnosis in breast cancer screening, from a randomized trial and from the Breast Screening Programme in England. J Med Screen 17: 25-30

Evans AJ, Pinder SE, Ellis IO, Wilson ARM (2001) Screen detected ductal carcinoma in situ (DCIS): over-diagnosis or an obligate precursor of invasive disease? J Med Screen 8: 149-151

Harris JR, Morrow M (2009) Clinical Dilemma of ductal carcinoma in situ. J Clin Oncol 27: 53303-55305

Jørgensen KJ, Gøtzsche PC (2009) Overdiagnosis in publicly organised mammography screening programmes: systematic review of incidence trends. BMJ 339: b2587doi:10.1136/bmj.b2587

Jørgensen KJ, Gøtzsche PC (2010) Who evaluates public health programmes? A review of the NHS Breast Screening Programme. J R Soc Med 103: 14-20

Joslyn SA (2006) Ductal carcinoma in situ: trends in geographic, temporal, and demographic patterns of care and survival. Breast J 12: 20-27

Kerlikowske K, Molinaro A, Cha I, Ljung BM, Ernster VL, Stewart K, Chew K, Moore DH 2nd, Waldman F (2003) Characteristics associated with recurrence among women with ductal carcinoma in situ treated by lumpectomy. J Natl Cancer Inst 95: 1692-1702

Lambert PC, Thompson JR, Weston CL, Dickman PW. (2007) Estimating and modeling the cure fraction in population-based cancer survival analysis. Biostatistics 8: 576-594

Lee LA, Silverstein MJ, Chung CT, Macdonald H, Sanhavi P, Epstein M, Holmes DR, Silberman H, Ye W, Lagios MD (2006) Breast cancer-specific mortality after invasive local recurrence in patients with ductal carcinoma-in-situ of the breast. Am J Surg 192: 416-419

Meijnen P, Peterson JL, Antonini N, Rutgers EJ, van de Vijver MJ (2008) Immunohistochemical categorisation of ductal carcinoma in situ of the breast. Br J Cancer 98: 137-142

Mokbel K, Cutuli B (2006) Heterogeneity of ductal carcinoma in situ and its effects on management. Lancet Oncol 7: 756-765

Morrow M, Strom EA, Bassett LW, Dershaw DD, Fowble B, Harris JR, O’Malley F, Schnitt SJ, Singletary SE, Winchester DP (2002) Standard for the management of ductal carcinoma in situ of the breast (DCIS). Cancer J Clin 52: 256-276

Nakamura S, Woo C, Silberman JH, Streeter OE, Lewinsky BS, Silverstein MJ (2002) Breast conserving therapy for ductal carcinoma in situ: a 20-year experience with excision plus radiation therapy. Am J Surgery 184: 403-409

National Cancer Intelligence Network and NHS Cancer Screening Programmes (2009) All breast cancer report: a UK analysis of all symptomatic and screen-detected breast cancers diagnosed in 2006 Sheffield, http://www.ncin.org.uk/search/all + breast + cancer + report.aspx (accessed 11 February 2011)

NHSBSP \& ABS (2010) An Audit of Screen Detected Breast Cancers for the year of screening April 2008 to March 2009, http://www.cancerscreening.nhs.uk/ breastscreen/publications/baso2008-2009.pdf (accessed 11 February 2011)

Office for National Statistics (1998) Cancer statistic registrations of cancers diagnosed 1971-1997, in England. Department of Health: London

Pinder SE, Duggan C, Ellis IO, Cuzick J, Forbes JF, Bishop H, Fentiman IS, George WD (2010) on behalf of the UK Coordinating Committee on Cancer Research (UKCCCR) Ductal Carcinoma In Situ (DCIS) Working party. A new pathological system for grading DCIS with improved prediction of local recurrence: results from the UKCCCR/ANZ DCIS trial. Brit J 103: 94-100

Sanders ME, Schuyler PA, Dupont WD, Page DL (2005) The natural history of low-grade ductal carcinoma in situ of the breast in women treated by biopsy only revealed over 30 years of long-term follow-up. Cancer 103: 2481-2484

Silverstein MJ, Lagios MD, Craig PH, Waisman JR, Lewinsky BS, Colburn WJ, Poller DN (1996) A prognostic index for ductal carcinoma in situ of the breast. Cancer 77: 2267-2274

Solin LJ, Fourquet A, Vicini F, Haffty B, Taylor M, Mccormick B, Mcneese M, Pierce LJ, Landmann C, Olivotto IO, Borger J, De La Rochefordiere A, Schultz DJ (2001) Mammographically detected ductal carcinoma in situ of the breast treated with breast-conserving surgery and definitive breast irradiation long-term outcome and prognostic significance of patient age and margin status. Int J Radiat Oncol Biol Phys 50: $991-1002$

Solin LJ, Kurtz J, Fourquet A, Amalric R, Recht A, Bornstein BA, Kiske R, Taylor M, Barrett W, Fowble B, Haffty B, Schultz DJ, Yeh IT, 
McCormack B, McNeese M (1996) Fifteen-year results of breast conserving surgery and definitive breast irradiation for the treatment of ductal carcinoma in situ of the breast. J Clin Oncol 14: 754-763

Tuttle TM, Jarosek S, Habermann EB, Arrington A, Abraham A, Morris TJ, Vernig BA (2009) Increasing rates of contralateral prophylactic mastectomy among patients with ductal carcinoma in situ. J Clin Oncol 27: 1362-1367

Virnig BA, Tuttle TM, Shamliyan T, Kane RL (2010) Ductal carcinoma in situ of the breast: a systematic review of incidence, treatment and outcome. J Natl Cancer Inst 102: 170-178
Wapnir IL, Dignam JJ, Fisher B, Mamounas EP, Anderson SJ, Julian TB, Land SR, Margolese RG, Swain SM, Constantino JP, Wolmark N (2011) Long-term outcomes of invasive ipsilateral breast tumour recurrences after lumpectomy in NSABP B-17 and B-24 randomised clinical trials for DCIS. J Natl Cancer Inst 103: 478-488

Welch HG, Woloshin S, Schwartz LM (2008) The sea of uncertainty surrounding ductal carcinoma in situ - the price of screening mammography. J Natl Cancer Inst 100: 228-229

Zahl PH, Strand HB, Mæhlen J (2004) Incidence of breast cancer in Norway and Sweden during introduction of nationwide screening: prospective cohort study. BMJ 328: 921-924

This work is published under the standard license to publish agreement. After 12 months the work will become freely available and the license terms will switch to a Creative Commons Attribution-NonCommercial-Share Alike 3.0 Unported License. 